\title{
Estudios de los efectos sociales de la construcción de represas hidroeléctricas y la conformación de redes científicas en América Latina (1992-2004)
}

\section{María Rosa Catullo}

Universidad Nacional de La Plata Argentina mrcatullo@ciudad com ar 


\section{Resumen}

En este articulo expongo en forma resumida la creacion de una red de antropologos sociales y cientificos sociales de America Latina en particular de Argentına y Brasıl que analizamos desde hace mas de una o dos decadas los efectos sociales de la construccion de represas hidroelectricas y la forma en que esta red nos ayudo en nuestro crecumuento personal y profesional

Palabras Claves grandes proyectos red Mercosur America Latina

\begin{abstract}
In this paper I describe the constitution of a network of Latin American soctal anthropologist and social scientists in particular those of Argentina and Brazll who have analyzed for more than two decades the social effects of the construction of hydroelectric dams and how this network has contributed to personal and professional growth
\end{abstract}

Keywords large projects networks Mercosur Latmamerica 
$\mathrm{E}$

n este trabajo, mi objetivo central es exponer sinteticamente la conformacion de una red de antropologos que analızamos desde hace más de una decada o dos los efectos sociales de la construcción de represas hidroeléctricas, y en particular, de las relocalızaciones compulsıvas de poblaciones urbanas o rurales (ındıgenas y campesı nos) Ademas, expondre como esta red permitió el constante dialogo y aprendızaje entre colegas argentmos y brasıleños Los "pıoneros" fueron Sílvı Coelho dos Santos (Unıversıdade Federal de Santa Catarina, Brasil) y Leopoldo J Bartolome (Unıversıdad Nacional de Misiones, Argentına), quienes ya mantenıan una relación laboral dado que el Dr dos Santos ya trabajaba en poblaciones indígenas afectadas por usınas hıdroeléctricas en el sur de Brasıl y el dr Bartolome realızaba estudıos en la represa bınacional argentıno-paraguaya de Yacyretá

$Y$, ¿Cual fue mi recorrido hasta llegar a integrar dicha RED? En ese sentıdo, me referıré a mi experıencia de trabajo de campo desde el año de 1979 hasta la fecha, al trabajo conjunto y el diálogo con científicos sociales y antropologos sociales argentınos, latınoamericanos y en partıcular, brasıleños, de la Unıversıdad Federal de Santa Catarına

En primer lugar, destaco mi expenencia de trabajo de campo en Argentina Como consecuencia de la construccion de la represa binacional argentıno-uruguaya de Salto Grande, se inundaron areas rurales, ası como tambien, el 70\% de la ciudad de Federación (margen argentına), y la pequeña ciudad de Villa Constitucion en la margen uruguaya Así, he trabajado en forma contunua (long term research) durante 29 años (de 1979 - a la actualidad) en lo que he denominado Vieja Federacıon Nueva Federación -Remanente Vieja Federación ${ }^{1}$ Inıcialmente, como auxiliar de investigacion, y en forma sistematica, desde 1980 como becaria de la Unıversidad Nacional de La Plata (1980-1983) y del Consejo Nacional de Investıgaciones Cientıfıcas y Técnicas (CONICET, 1984-I990), bajo la direccion del Dr Leopoldo J Bartolome

En lo que respecta al diálogo con colegas de otras areas de las Ciencıas Sociales, y colegas brasıleños, la partıcıpacıón en el $2^{\circ}$ Congreso Argentıno de Antropología, realızado en el año de 1986 en la ciudad de Buenos Aures, me permitio conocer y conversar con el entonces Magister Gustavo Lins Ribeiro, quien realızaba su trabajo de campo 
para su dısertación doctoral Con el compartí una Mesa Redonda sobre el "frustrado" proyecto de relocalización de la capital argentina a la ciudad de Viedma, provincia de Buenos Aires y posteriormente se convertiría en mi Director de tesis Doctoral

En 1990, con una beca del gobıerno de Brasıl (Coordınación de Perfeccionamiento de Personal de Nivel Superıor, CAPES) inicie el "Doctorado Conjunto Facultad Latinoamencana de Ciencias Sociales (FLACSO)-Universidade de Brasılia (UnB) en Estudıos Comparatıvos sobre América Latina y el Carıbe', en la cuudad de Brasılıa, bajo la orıentación del Dr Gustavo Lins Rubeiro Comenzaba una etapa de constante dıálogo, no solo con antropólogos sociales y cientıficos sociales (sociologos, economistas, politologos) de Brasil, sino de toda América Latına, tales como Luss de Castro Faria (UFRJ), Roberto Cardoso de Olıveira (UNICAMP), Sílvıo Coelho dos Santos (UFSC), Alıcia Barabas y Miguel A Bartolomé (INAH, Mexico), Nelly Arvello Jiménez (Instıtuto Nacional de Antropologia, Venezuela), Geralda Diaz Aparecida (UnB), Cristovam Buarque (UnB) Ademas, esta etapa también estuvo marcada por el análısıs profundo de gran parte de la bıblıografıa exıstente (argentına, brasıleña mexıcana, uruguaya) sobre los efectos socıales de las represas hidroeléctricas, y de la documentación sobre la resistencia de los afectados, quienes se organizaron en la Comissão Regional de Atıngidos por Barragens (CRAB) $y$ en el Movimento Nacional de Atıngıdos por Barragens (MNAB O MAB) Asımısmo, se estudio la documentacion de determinados entes, tales como la Eletrobras, Eletrosul (Brasil) y la Comısıón Técnıca Mixta de Salto Grande (argentina y uruguaya)

En noviembre de 1992 tuve la oportunıdad de ser invitada por la Asocıação Brasıletra de Antropologıa (ABA), y partıcıpé del Workshop "Polltıcas Publıcas e Populações Locats, realızado en la Unıversıdad Federal de Santa Catarına (UFSC), donde se inıcıó un dıalogo muy merıtorıo para mı formación, con el Prof Dr Sílvıo Coelho dos Santos, investıgador y Director del Nucleo de Pesquisas de Populações Indigenas (NEPI), y con investıgadoras del Programa de Pos graduação em Antropologıa Socıal de la UFSC, tales como, las profesoras Ilse Sherrer Warren, María José Reıs, Neusa Bloemer y la Prof Ceclla Helm de la Unuversıdad Federal de Parana (UFPR) Esta reunion marcarıa el comıenzo de un dialogo y de 
trabajo con los colegas de la Universidad Federal de Santa Catarina

En el año de 1993 realice trabajo de campo durante 9 meses (etnografía multulocal) en la cuudad de Buenos Aures, la crudad de Parana (capital de la provincia de Entre Rıos), la ciudad de Concordía y en la cludad Nueva Federación (inundada y relocalızada por la construcción de la represa bınacional argentıno-uruguaya de Salto Grande) En lo que respecta a Uruguay, estuve recabando información en Montevideo(capital de Uruguay), en la ciudad de Salto y en Villa Constıtucion, pequeña ciudad ınundada y relocalızada parcialmente, tambien por la represa de Salto Grande Por ultumo, analıce documentacion y realıcé entrevistas a empleados, tecnicos y funcionarıos ${ }^{2}$ de las Delegaciones Argentuna y Uruguaya de la Comision Tecnuca Mixta de Salto Grande Defendı mı Tesis Doctoral en mayo de 1996

Cuando regrese a Argentına, a partır de 1996 y hasta el año 2004, he particıpado y coordınado, como profesora e investıgadora de la Unıveısıdad Nacıonal de La Plata, diferentes proyectos de investıgacion, junto a Silvio Coelho dos Santos (UFSC), como coordınador brasıleño El prımero de estos proyectos “Partıcıpacıon y Resıstencia en Proyectos de Gran Escala Analısıs comparatıvo de Represas Hıdroelectricas en Argentına Brasıl Y Uruguay" (1996 1998) fue subsıdıado en 1997 por la Fundación Antorchas (Argentına), y en 1998, por la Coordenação e Apeferçoamento de Pessoal de Nivel Superior (CAPES Brasil) Partıcıparon, ademas, del prof Sílvıo C Dos Santos, integrantes del NEPI la prof Cecılıa Marıa Vieıra Helm - investıgadora del CNPq/ UFPR- y la prof Marıa José Reıs

En el marco de esta investigacion, el prof Silvio Coelho dos Santos visito el mes de febrero de 1997 la catedra de Antropología Sociocultural II (FCNYM-UNLP) donde me desempeñaba como profesora y postenormente, viajo a la ciudad de Posadas, donde dio una conferencia en el Posgrado en Antropologia Social (Universidad Nacional de Misiones), donde el dr Bartolome se desempeña como Director En la ciudad de La Plata, el prof Silvio C dos Santos, quien desde la década del ' 80 desarrollo estudıos sobre los efectos sociales en poblaciones indigenas por la realızacion de represas hidroelectricas y era Coordınador en la UFSC del Proyecto "Indıos e barragens no Sul do Brasıl", efectuo una visita a la ciudad de La Plata, en que realızo 
conferencias, de las que participaron graduados y alumnos, integrantes del equipo que coordınaba quien suscribe

En los encuentros se analizaron diversos y relevantes temas como (a) la política del sector electrico de Brasil (Eletrobras, Eletrosul) y su comparacion con las politicas en Argentina y Uruguay, (b) la confor macion de la $C R A B$ (Erexim 1979) y del (MNAB), sus politıcas y el rol que ocuparon en su formación y en su postenor accionar la Iglesıa ( $\mathrm{g}$ Catolıca y Evangelısta), los partıdos politıcos (vg , Partıdo dos Trabalhistas $(P T)$ ) y los sindicatos nucleados en la Central Unica de Trabalhadores (CUT) - tematica que también abordaria la prof María J Reıs, y un tema que merece ser destacado es (c) el analısıs de las diferentes posturas que un antropologo puede tener ante los Proyectos de Gran Escala entendidos por los entes gubernamentales y entes multilaterales de financiacion como "proyectos de desarrollo" y la etica del antropologo

Dada la trayectoria del prof dos Santos, su aporte en este tema fue muy productivo ya que nos planteo que estas tematicas proponen el dulema de como actuar ante los requerımientos de asesona o consultoria por parte de los entes gubernamentales y/o internacionales de financiación ( $\mathrm{vg}$, World Bank, BID), con el fin de evaluar las consecuencias sociales de la construcción de estos Proyectos de Gran Escala Es decir ya dos Santos planteaba una 'antropologia de accion" basada en su experıencıa, aunque para tales funes debamos lıberarnos de las tradicionales connotaciones de nuestra disciplina (por ejemplo, Antropologia Aplicada) ¿Cuál es el compromıso del antropologo frente a estos grandes emprendımıentos, frente a los actores colectıvos con mayor poder (vg entes, estructuras gubernamentales, compañías contratıstas) y frente a las poblaciones afectadas? En este sentıdo, se remarcó la capacıdad critıca y relatıvızadora de la Antropologıa Además, se destacó el papel de broker del antropologo entre los diferentes actores colectıvos, y la importancia de que el antropólogo asesore desde el ınıcıo del PGE y de los reasentamientos no solo a los entes gubernamentales, sıno también a los propıos movimientos de las poblaciones afectadas

Por el mismo Convenıo, vinieron a la ciudad de La Plata, la prof Ceclıa Marıa Vieıra Helm - investıgadora del CNPq/ UFPR - y la prof María José Reıs quienes partıcıparon del Grupo de Trabajo "Problemas socioambientales movimientos poblacionales y relocalızaciones 
coordınado por mu, dentro del marco del VII CAAS (julo de 1997) Asimismo junto al dr Leopoldo J Bartolome, el dr Alejandro Balazote (Unıversidad de Buenos Aires UNCPBA) y el dr Juan Carlos Radovich (Universidad de Buenos Aures Instituto Nacional de Antropologia y Pensamiento Latınoamencano) intervinieron en el Panel-Tema "Poder y Partıcipacion en Proyectos de Gran Escala Analısıs Comparatıvo de Represas Hıdroeléctricas de Argentına y Brasıl", coordınado por mí (01/08/1997), donde analizamos comparatıvamente, a partır de las experıencıas de investıgación de los partıcıpantes las respuestas de las poblaciones afectadas por la construccion de represas hidroelectricas en Argentina y Brasil, y buscamos comprender el marco cultural, socıoeconomico, polítıco y jurıdıco en que se dieron las diferentes prácticas de estos actores sociales Ya este Convenıo nos permitıó relacionarnos con el dr A Balazotte y el dr J C Radovich quienes se desempeñan como profesores e investıgadores de la Unıversıdad de Buenos Aıres (UBA), de la Unıversidad Nacional del Centro de la Provincia de Buenos Aures (UNCPBA, Olavarria) y el Instıtuto Nacional de Antropologia y Pensamiento Latinoamericano (INAPL)

Esta partıcipacion tuvo una doble sıgnificación Prımero, por los aportes de ambos investıgadores referentes a la construcción y consecuencias socioambientales de las represas construidas en el norte de la Patagonia (Argentina) y por el hecho de formar una RED de antropólogos sociales argentunos y brasıleños

Consideramos importante destacar que el proyecto en su totalidad, posibilitó, además de la cooperacion entre investıgadores de ambos passes, la formacion de ıecursos humanos, tanto en las sedes argentınas, como en la brasıleña

Paralelamente, esta red argentıno-brasıleña de antropologos "barragetros, continuo trabajando en conjunto y en el mes de noviembre de 1997, integrantes de ambos equipos participamos de la II Reunion de Antropología del Mercosur, (Pırıapolıs, Uruguay), en el Grupo de Trabajo "La implantacion de hidroelectricas y sus consecuencias sociales", que coordinaron los dres Bartolome y dos Santos Y en el año de I998 prosıguió sus dialogos en la XIX Reunion de la Asociacion Brasileña de Antropología, donde las profesoras Helm y quien suscribe coordinaron el Grupo de Trabajo Cultura, Poder e 
Medio Ambiente as usınas hıdreletrıcas e as populações locaıs na Argentına Uruguay e Brasil" (Vitorıa, Brasıl)

En el año 1999, comenzó una etapa nueva, pues ya comenzaron los trabajos de campo realızados por la dra Reis y mi persona a la ciudad de Ita (Estado de Santa Catarına), relocalızada por la construcción de la represa homónıma (Estado de Santa Catarma y Rio Grande del Sur) al ıgual que áreas rurales, tematıca central de la Tesis Doctoral de la prof Reıs (Cf, 1998) En dicho viaje, aprovechamos la cercanıa, y estuvimos también en la sede de la CRAB, en la ciudad de Erexim (RS), donde entrevistamos a sus líderes, y a los afectados por la represa de Ita, de otras pequeñas ciudades

La últuma etapa (2001-2004), se realızo con Convenıs Internacionales sucesivos de la Coordenação e Apeferçoamento de Pessoal de Nivel Superior (CAPES Brasil) y la Secretaria para la Tecnologia, la Ciencia y la Innovación Productıva (SeTCIP, Argentına), y el proyecto se tıtuló "Poder y Proyectos de Desarrollo cuestiones politicas y socioculturales y nuevos interlocutores en el escenario del Mercosur" Es interesante destacar que se sumaron al Convenıo, el dr Alejandro Balazote, el dr Juan Carlos Radovich y el entonces Mgter (hoy doctorado) Omar Arach (PPGAS-UNaM) por Argentına, en tanto por Brasıl, ingresaron la dra Alıcia G de Castells y la dra Neusa Bloemer (UFSC)

Y posteriormente en la IV Reunıon de Antropología del Mercosur (RAM) realızada en la ciudad de Curitıba, en el año 2001, comenzamos un fructífero dialogo con la Magıster Marılın Rehnfeldt, profesora e ınvestıgadora de la Pontıficıa Universıdad Católıca de Asunción Paraguay, o sea, una nueva integrante de esta "red '

En estos años, las actıvidades fueron diversas y diferentes Por un lado, se contınuó con los Semunarıos, Mesas Redondas y Workshops realızados en la Unıversıdad Federal de Santa Catarına (años 2001 y 2003) en la Universıdad de La Plata (año 2002) y en el Instıtuto Nacıonal de Antropología y Pensamiento Latınoamericano (INAPL, año 2004) Paralelamente, realızamos nuevos trabajos de campo en la cludad de Ita con la Dra Reıs y en el año 2003 la dra A G De Castells también paso a formar parte de los trabajos Ambas investigadoras estuvieron en la cuudad de Federacion (Argentına), ınundada y relocalızada por la represa de Salto Grande (años 2002 y 2003) 
Estas experıencias nos permitıeron analızar y comparar las diferentes políticas gubernamentales (nacionales, provmciales/estaduales y locales) y las practicas de los actores sociales involucrados en los procesos de relocalızación, las cuales demostraron grandes diferencras, como por ejemplo la postura a favor de las represas - tanto en las cludades de Federación como de Itá -, y de absoluta resistencia en las áreas rurales del sur de Brasıl -lo cual dıo lugar a la formacıon de la CRAB y luego, del MAB

La conformacion de esta 'red de barrageıros" de Argentına, Brasıl - en particular con los colegas de la UFSC - y Paraguay se fue ampliando con el correr de los años porque cada uno de los integrantes hemos conocido y trabajado con otros colegas En mi caso, he trabajado con el dr Scott Robınson de la Unıversidad Autónoma de Iztapalapa (Mexico), el dr Gustavo Castro Soto (Chuapas, Mexico) y el dr Alberto Garcia (Unı versıdad de Queretaro Méxıco) y con otros colegas brasıleños, como el dr Franklın Rothman (Unıversıdad Federal de Vıçosa), el dr Parry Scott (Unuversıdad Federal de Pernambuco), , la dra Edna Castro, el dr Carlos A Magalhaes, la prof Sonıa Magallhaes (Unıversıdad Federal de Pará), la dra Guomar Germanı (Unıversıdad Federal de Bahıa) e integrantes del INPPUR Unıversıdad Federal de Rıo de Janeiro

Esto ha significado una posibilıdad de contınuo enriquecimiento y crecimiento individual y grupal, el cual quedó expuesto en una vasta produccion cientifica libros (indıviduales o complaciones), disertaciones de posgrado, articulos en revistas y libros (consultar biblıografía) Y tambien hemos podido demostrar que se puede trabajar en conjunto

Lo más importante, a mi criterıo, es que esa red de científicos sociales pudo conformar una "red de solıdarıdad" donde se podıa recurrir a un "otro/a" a pesar de las distancias y contando con la tecnologia Hoy, ya en camunos diferentes, las distancias se notan y en lo personal, siento saudades de los Proyectos conjuntos, pero se que nos encontraremos en un Congreso o unas Jornadas y podremos dar vida de nuevo a esa red, que allá por 1997 inıcié junto a Sílvio C dos Santos

\section{Notas}

1 Denomino ciudad de Federacton al antıguo asentamiento mundado en el año 1979 y Nueva Federacion a la actual cudad de acuerdo a los propios habitantes Sin 
embargo en el caso de la cuudad de Ita los itaenses continuan denominando tanto al anterior asentamiento como a la actual localızacion como ciudad de Ita

2 En Argentina funcionarıo es aquel empleado de caracter politico es decir que cambia de acuerdo al gobierno sea local provincial o nacional

\section{Bibliografia}

ARACH Omaı La lucha sobre el rio un analisis de la oposicion a la represa del Parana Medio (Entre Rios 1996 1997) Tesıs (Maestria en Antıopologia Socıal) - Programa de Postgrado en Antropologıa Socıal Unıversıdad Na cional de Misiones Posadas 1999a

ARACH Omar La represa y el drama conflictos sociales y argumentos am bientalistas en torno a la represa del Parana Medıo Revista POS Instituto de Ciêncıas Socıaıs Unıversıdade de Brasılıa Brasılıa p 217232 1999b ARACH Omai Entre rios si/entre represas no la partıcipacion ambienta lista en la oposicion a la represa del Parana Medio In BALAZOTE A CATULLO M R RADOVICH J C (Org) Antropologia y grandes proyectos en el Mercosur La Plata Minerva 2001 p 187212

ARACH Omar Ambientalismo desarrollo y transnacionalidad en Paraguay con sideraciones a partir de las protestas sociales en relacion a Yacyreta Bue nos Aures IDES 2002 (Cuadernos para el Debate 16)

ARACH Omar Ambientalismo desarrollo y transnacionalidad en Paraguay consideraciones a paitır de las protestas sociales en relacion a Yacyreta 1n JELIN Elizabeth (Comp) Mas alla de la nacion las escalas multiples de los movimientos sociales Buenos Aures Libios de Zorzal 2003a p 105159

ARACH Omar Ambientalısmo proyectos de gran escala y transnacionalı dad el caso de la Coalicion Rios Vivos y la oposicion a la Hidrovia del Mercosur Revista AVA Programa de Posgrado en Antıopologıa Social Universidad Nacional de Misiones Posadas p $35522003 \mathrm{~b}$

ARACH Omar Rıo revuelto desempeño y trayectorıa de una organızacıon ambientalista paraguaya en la oposicion al pioyecto hidroelectrico Ya cyreta (1991 2000) Disertacion (Doctorado en Antropologia Social) Programa de Postgrado en Antropologıa Social Unıversıdad Nacional de Misiones Posadas 2006

BALAZOTE, Alejandro Efectos socioambientales de la explotacion gasifera y petiolfera en la cuenca neuquina Cuadernos de Antropologia Social Instituto de Ciencias Antıopologicas Facultad de Filosofia y Letras UBA n 11 2001a

BALAZOTE Alejandro Explotacion hidrocarburifera y conflicto social en Runcon de los Sauces In BALAzOTE Alejandro CATULLO Maria Rosa RADOVICH Juan Carlos (Org) Antropologia y giandes proyectos en el Merco sur La Plata Minerva 200lb p 147161

BALAZOTE Alejandro Derıames petrolıferos impacto social en localidades del no Colorado Anuario de la Escuela de Historia Facultad de Filosofia y Humanidades Unıversidad Nacional de Cordoba v 2 n 22002

\section{ILHA}


BALAZOTE Alejandro Reasentamiento forzoso de poblacion y regularızacion territorial en el Interfluvio Teuco Bermejito (Provincia de Chaco) Cuadernos de Antropologia Social Facultad de Filosofia y Letras, UBA n 152003

BALAZOTE Alejandro CATULLO Maria Rosa RADOVICH Juan Carlos (Comp ) Antropologia social de grandes proyectos en el Mercosur La Plata Mu nerva 200la

BALAZOTE Alejandro CATULLO Marıa Rosa RADOVICH Juan Carlos Introduccion In BALAZOTE, A CATULLO $M$ R RADOVICH $\mathrm{J} C$ (Comp ) Antropologia y grandes proyectos en el Mercosur La Plata Minerva $2001 b$

BALAZOTE Alejandro RADOVICH Juan Carlos Effects of megaprojects on an Indigenous reserve in North Patagonia (Argentina) In INSTITUT FUR VOLKERKUNDE COMMITTEE S SECRETARIAT Bulletin of the In ternational Committee on Urgent Anthropological and Ethnological Research Viena 1995/96 International Union of Anthropological and Ethnologi cal Sciences (IUAES)

BALAZOTE A, RADOVICH J C Loma la Lata los inconvenientes de vivir sobre el gas Nosotros los Otros Facultad de Flosofia y Letras UBA $\vee 1 \mathrm{n}$ 2 p I2 I3 oct 1997

BALAZOTE A RADOVICH $\mathrm{J}$ Se nos declaro la muerte efectos socioam bientales de la explotacion de hidrocarburos en la agrupacion Painemil In PEREZ ARIAS Enrique (Ed) La reconstruccion del mundo en America Latina Lund Sweden Heterogenesis 1998 v 2

BALAZOTE A RADOVICH $\mathbf{J} \mathbf{C}$ Inversion de capital y riesgo en grupos mapuche asentados en el yacimiento de Loma La Lata In TRINCHERO Hector $\mathrm{H}$, BALAZOTE Alejandro O (Comp) Etnicidades y territorios en re definicion perspectiva historica y antropologica Buenos Aires Secretaria de Ciencia y Tecnica Facultad de Humanidades 2000 (Estudıos desde la Reahdad Argentına)

BALAZOTE Alejandro RADOVICH J C El proceso de privatızacion de Y PF (Yacimientos Petrolıferos Fiscales) Cuadernos del INAPL Instituto Nacio nal de Antropologia y Pensamiento Latınoamericano Buenos Aures n 192002

BALAZOTE Alejandro RADOVICH Juan Carlos Grandes represas hidroelectricas efectos sociales sobre poblaciones Mapuches en la Region del Comahue Argentina In SANTOS Silvio Coelho dos NACKE Ane hese (Org ) Hidreletricas e povos indigenas Florianopolis Letras Contempo râneas 2003 p 85110

BALAZOTE Alejandro RADOVICH $\mathrm{J}$ C Efectos sociales de la privatizacion de YPF en la provincia de Neuquen Cuadernos de Antropologia Instituto Nacional de Antropologia y Pensamiento Latınoamericano Buenos Aires n Il 2003b

BALAZOTE A RADOVICH $\mathrm{J}$ C A treinta años del reasentamiento de $\mathrm{P} 1$ cun Leufu Cuadernos del INAPL Instituto Nacional de Antropologia y Pen samiento Latmoamericano Buenos Aures n 202007 
BARTOLOMÉ Leopoldo $J$ The colonos of Apostoles adaptative strategy and ethnicity in a Polısh Ukrainian settlement in Northeast Argentina New York AMS Press 1991

BARTOLOME Leopoldo J LUKASZ Danuta STEMPLOWSKI Ryszard Slowianie $w$ argentynskım Misiones 18971977 Warsaw Panstwowe Wydawnietwo Naukowe 1991

BARTOLOME Leopoldo J El extranjero profesional y la tentacion faustica la antropologia frente a los proyectos de desarrollo In ARANTES A A RUBEN G DEBERT G (Comp) Antropologia e direitos humanos a res ponsabilidade do antropologo Campinas Brasıl Ed da Unicamp 1992 p 109132

BARTOLOME Leopoldo J The Yacyreta expenence with urban resettlement some lessons and insights In CERNEA Michael $M$ GUGGENHEIM Scott (Comp ) Anthropological approaches to resettlement policy practice and theory Boulder CO Westview Press 1993 p 109132

BARTOLOME Leopoldo $J$ Theorical and operational issues in resettlement processes the Yacyreta Project and urban relocations in Posadas (Argen tuna) In UNITED NATIONS CENTRE FOR REGIONAL DEVELOPMENT (UNCRD) (Ed) Environmental and social dimensions of reservoir development and management in the La Plata River Basin Nagoya Japon UNCRD 1994 p 4357 (Research Report Senes 4)

BARTOLOME Leopoldo $\mathrm{J}$ Impactos sociales del desplazamiento poblacio nal trascendiendo el modelo etnografico Estudios Regionales Secretarı de Investigacion FHyCS UnaM \& 8 p 56611995

BARTOLOME Leopoldo Jose Impactos sociales del desplazamiento pobla cional trascendıendo el modelo etnografico In MAGALHÃES S BRIT TO E de C CASTRO E R (Org) Energıa na Amazônı Belem Museu Paraense Emılıo Goeldı Unıversıdade Federal de Para Assocıação das Unıversıdades Amazônıcas 1996 v l p 431438

BARTOLOME Leopoldo Jose Combatiendo a Leviatan la artıculacion y d fusion de los movimientos de oposicion a los proyectos de desarrollo hi droelectrico en Brasil (1985 91) Desarrollo Economico IDES Buenos Aires v 39 n 153 p 771021999

BARTOLOME Leopoldo Jose GPDs y desplazamientos poblacionales algu nas claves para su comprension como procesos sociales complejos AVA Revista de Antropologia Social Universidad Nacional de Misıones Posadas v l n 2 p 11202000

BARTOLOME Leopoldo Jose Combatıendo a Leviatan la artıculacion y difusion de los movimientos de oposicion a los proyectos de desarrollo hidroelectrico en Brasil (1985 91) In BALAZOTE Alejandro CATULLO Maria Rosa RADOVICH Jorge (Comp) Antropologia y grandes proyectos en el Mercosur La Plata Minerva 2001 p 1558

BARTOLOME Leopoldo Jose Population resettlement schemes as social processes conceptual and methodological issues In INTERNATIONAL SYMPOSIUM ON RESETTLEMENT AND SOCIAL DEVELOPMENT Ho 
Estudios de los efectos sociales de la construccion de represas hidroelectricas y la conformacion de redes cientificas en America Latına (1992 2004)

hal University Nanjıng China en mayo 12142002 Proceedings Nan jing 2002

BARTOLOME Leopoldo Jose Planned population resettlement as social processes ambiguities manipulations and agency" Comision Mundial de Recur sos Acuaticos 2009 En prensa

BARTOLOME Leopoldo Jose Fighting Leviathan the articulation and spread of local opposition to hydrodevelopment in Brazil In ANUAL LATIN AMERICAN STUDIES CONFERENCE 4I INVOLUNTARY MI GRATION AND RESETTLEMENT IN LATIN AMERICA Gainsville Flon da USA Proceedings Gainsville 2009 En prensa

BLOEMER Neusa Marıa Sens Brava gente brasıletra migrantes italianos e caboclos nos campos de Lages Flonanopolıs Cidade Futura 2000

BLOEMER Neusa Marıa Sens A hidreletrica de Campos Novos campone ses migração compulsoria e atuação do setor eletrıco In REIS Marıa Jose BLOEMER Neusa M Sens (Comp) Hidreletricas e populaçōes locais Florianopolis Cidade Futura Ed da UFSC 2001 p 93118

BLOEMER Neusa M Itınerâncias e mıgrações a reprodução social de peque nos produtores e as hidreletricas Tese (Doutorado em Antropologia So cıal) - Universıdade de São Paulo São Paulo 1996

BORNHOLDT Luciano Campelo Familıa rural e reprodução social estudo de caso da UHE Quebra Queixo Dissertação (Mestrado em Antropologia So cial) - Programa de Pos Graduação em Antropologıa Socıal Centro de Filosofıa e Ciêncıas Humanas Unıversıdade Federal de Santa Catarına Florianopolis 2003

CATULLO Maria Rosa Reconstruccion de la identıdad y proyectos de gran escala Ciudad Nueva Federacion Provincia de Entre Rios Argentına Brasılıa Departamento de Antropologıa Unıversıdade de Brasılıa 1992 (Sene Antropologia 125)

CATULlo Maria Rosa La antropologia y los proyectos de gran escala los estudıos sobre represas hidroelectrıcas en Brasıl Anuario Antropologıco Rıo de Janeiro Tempo Brasileiro n 90 p 205 229, 1993

CATULLO Maria Rosa A antropologia e as represas hidroeletricas no Bra sıl Cadernos da America Latına Centro de Pesquisa e Pos Graduação sobre Amenca Latına e o Carıbe (CEPPAC) Fundação Unıversıdade de Brasılıa Brasilia n 1 1996a

CATULLO Maria Rosa Poder y participacion en proyectos de gran escala analisis comparativo de los procesos de relocalizacion por la construccion de la represa binacional argentino uruguaya de Salto Grande Tese (Doutorado em Clêncıas Socıals) - Faculdade Latıno Amerıcana de Clências Sociaıs Unıversıdade de Brasilıa Brasilıa 1996b

CATULLO Marıa Rosa La antropologia del desarrollo un enfoque multı variado para el analisıs de la fundación de la ciudad de La Plata como proyecto de gran escala (PGE) In JORNADAS DE HISTORIA ARGENTI NA Y AMERICANA 2 El Fin de Siglo El Hombre v su Tiempo Universi dad Catolıca Argentına Buenos Arres Actas Buenos Arres 1998 
CATULlo Maria Rosa Proyectos de gran escala en el marco del Mercosur clases sociales intereses sectoriales y brokers en procesos de relocalizacion Cuadernos del INAPL Buenos Arres n 18 p 69901999

CATULLO Maria Rosa Grandes emprendimientos en el contexto del Merco sur centralismo poder regional y participacion ln CONGRESO ARGEN TINO DE ANTROPOLOGIA SOCIAL 6 Identıdad Disciplunana y Campos de Aplicacion Mar del Plata 2000 Actas Mar del Plata 2000

CATULLO Maria Rosa La represa de Salto Grande partıcipacion untereses sectoriales y nacionales In JORNADAS DE HISTORIA ARGENTINA Y AMERICANA, 3 Pontıficia Universıdad Catolica Argentına Buenos Ai res 2000 Actas Buenos Aures 2000b v 2 p 775785

CATULlO Maria Rosa Poder y niveles de decision en proyectos de gran escala In BALAZOTE A CATULLO $M$ R RADOVICH $J$ C (Org) Antropologia y grandes proyectos en $\mathrm{tl}$ Mercosur La Plata Minerva 200la $\mathrm{p}$ 131150

CATULLO Maria Rosa Centralismo poder regional y participacion la ciu dad de Federacion, Entre Rios Argentina In REIS Marıa Jose BLOE MER Neusa (Org) Populações locals projetos de desenvolvimento e questóes socio ambientais Florianopolis Ed da UFSC Futura 2001b p 167196

CATULLO Mara Rosa Estudios de impactos sociales en el Mercosur pro cesos relocalizatorios nuevos espacios urbanos y reconstruccion de redes de relaciones sociales Revista de Antropologia Social Revista de la Seccion Antropologia Social Facultad de Fulosotia y Letras Unıversıdad de Bue nos Aures n 15 16, p 49692002

CATULLO Maria Rosa Participacion articulacion y poder en proyectos de gran escala HABITUS Pontıficıa Unıversıdade Catolıca de Goıas Golânıa V l n l p 1812192003

CATULLO Mana Rosa Ciudades relocalızadas una murada desde la antropolo gia social Buenos Aures Biblos 2006

CATULLO Maria Rosa FERRARI, Lucrecia, MARTINEZ Lucas Proyectos de desarrollo identıdad y turismo termal Ciudad Nueva Federacion Entre Rios Argentina In REIS Maria Jose, BALAZOTE Alejandro RADOVI CH Juan Carlos (Ed) Disputas territonales y conflictos interetnicos en Brasil y Argentma Cordoba Ferreura 2005 p 185196

CATULLO Marıa Rosa PATTI Beatriz Proceso de relocalızacion y nueva cludad Federacion Nueva Federacion Entre Rios Argentına In CON GRESO ARGENTINO DE ANTROPOLOGIA SOCIAL 5 La Plata 1997 Actas La Plata 1997

CATULLO Marıa Rosa PATTI Beatriz Proceso de relocalızacion y nueva cludad Federacion Nueva Federacion Entre Rios Argentina In BALA ZOTE A CATULLO M R RADOVICH J C (Org) Antropologia y grandes proyectos en el Mercosur La Plata Mmerva 2001 p 109129

CATULLO Maria Rosa SHIMKO Susana Actores sociales e intereses secto riales en la fundacion de la cuudad de La Plata In JORNADAS DE HIS 
TORIA ARGENTINA Y AMERICANA 3 Pontıficia Universidad Catolica Argentuna Buenos Arres 2000 Actas Buenos Aures 2000 v 2 p 483 490

CATULLO Mana Rosa et al La cludad de La Plata primer proyecto de gran escala de la Argentina su fundacion sus cambios (siglos XIX XX) In JORNADAS DE HISTORIA ARGENTINA Y AMERICANA 2 EL FIN DE SIGLO EL HOMBRE Y SU TIEMPO Universidad Catoluca Argentına Buenos Aires 1998 Actas Buenos Arres 1998

CATULLO Maria Rosa REIS Marıa Jose CASTELlS Alıcia G de Patrı mônıo reassentamento compulsorıo e turısmo um estudo comparatıvo sobre Federacıon (Argentına) e Ita (Brasıl) In HERNÁNDEZ LLOSAS Isabel (Org) CONGRESO DE AMERICANISTAS 5I Argentuna Anales Buenos Alles 2003

HELM Cecilıa M Vieira Grandes projetos hidroeletricos e os povos indigenas Kain gang e Guarant no Estado de Parana Brastl In CONGRESO INTERNACIO NAL DE AMERICANISTAS 48 Estocolmo Suecia jul 1994 Mimeo

HELM Cecilıa M Vıeıra A justıça e lenta a FUNAI devagar e a pacıêncıa dos indıos esta se esgotando pericia antropologica na area indigena Man gueirmha PR Textos e Debates Laudos Periciais Antropologicos Nucleo de Estudos sobre Identıdade e Relações Interetnıcas Unıversıdade Federal de Santa Catarma v 2 n 4 p 22381996

HELM Cecílıa M Vieıra Estrategıas indigenas face a implantação de barragens em seus terrtorios estudo dos Kaingang e Guaranı da bacia do Rio Tibagı In CONGRESO ARGENTINO DE ANTROPOLOGIA SOCIAL 5 Unıversıdad Nacional de La Plata La Plata jul /agosto 1997a Mimeo

HELM Cecilia M Vieira Direitos territoriats indigenas disputa judicial entre Kangang Guaranı e madereıros pela Terra Indıgena Mangurinha Para na Brasil In CONGRESO INTERNACIONAL DE AMERICANISTAS 49 Quito jul 1997b Mimeo

HELM Cecilia M Vieira Laudo antropologico sobre os povos indigenas da Bacıa do Rio Tibagl os Kanngang e os Guaranı Curitiba Copel 1998

HELM Cecilia M Vieira Laudo antropologico sobre os povos indigenas da Bacia do Rio Tibagı Curıtiba Copel I999a

HELM Cecilıa M Viena Kaingang e Guarant das terras da Bacta do Rio Tibagı usinas hidreletricas e seus impactos Curitiba Ed da UFP 1999b

HELM Cecilıa M Vieira Kaingang e Guaranı da terra indigena Mangueı nunha e a usina hıdreletrica Salto Santıago no Rıo Iguaçu (PR) REIS Marıa Jose BLOEMER Neusa Marıa Sens (Org) Hidreletricas e populaçōes locals Flonanopolis Cidade Futura Ed da UFSC 200la p 3970

HELM Cecilıa M Vieira Povos indigenas e projetos hidreletricos no Rio Ti bagı In BALAZOTE Alejandro CATULLO Marıa R RADOVICH Juan Carlos (Org ) Antropologia y grandes proyectos en el Mercosur La Plata Miner va $2001 \mathrm{~b}$ p 6988

HELM Ceciha M Vieira A consulta aos indios da Bacia do Rio Tibagı PR a usına hidreletrica São Jerônumo e a questão etıca In SANTOS Sılvıo 
Coelho dos NACKE Aneliese (Org) Hidreletricas e povos indigenas Flona nopolıs Letras Contemporâneas 2003 p III I34

HELM Cecilia M Vieira (Coord) SANTOS, Silvio Coelho dos NACKE Aneliese A implantação de ustnas hidreletricas e os indigenas no Sul do Brastl Curıtıba SEMA 1996 Trabalho produzıdo para o Instituto Ambiental do Parana Mimeo

PATTI Beatriz CATULLO Maria Rosa Proceso de relocalizacion y nueva ciudad Federacion Nueva Federacion Entre Rios Argentina In BALA ZOTE Alejandro CATULLO Maria R RADOVICH Juan Carlos (Org) Antropologia y grandes proyectos en el Mercosur La Plata Minerva 2001 p 107126

RADOVICH Juan Carlos El proceso de privatizacion de Hidronor S A In BALAZOTE Alejandro CATULLO Maria Rosa RADOVICH Juan Carlos (Org) Antropologia y giandes proyectos en el Mercosur La Plata Minerva 2001 p 163186

RADOVICH Juan Carlos El impacto social de las grandes represas Nove dades de Antropologia INAPL Buenos Aures v 10 n 38 p $34200 \mathrm{la}^{\mathrm{a}}$ Parte

RADOVICH Juan Carlos El impacto social de las grandes represas antece dentes sobre relocalızaciones de pueblos indigenas Novedades de Antropolo gla INAPL Buenos Arres v $10 \mathrm{n} 39$ p 1822 200 lb 2a Parte

RADOVICH Juan Carlos El impacto social de las grandes represas el deba te en torno a las represas Novedades de Antropologia INAPL Buenos Aues v 10 n 40 p 1214 200lc Ultıma Parte

RADOVICH Juan Carlos Impacto soctal de grandes aprovechamtentos hidroener getzcos sobre comunidades rurales de Norpatagonta Tesis (Doctoral) - Facultad de Filosofia y letras Universidad de Buenos Aıres 2003

RADOVICH $J$ BALAZOTE, A Hasta el no cambio de color impacto social y re localızacion de poblacion en Casa de Piedra (Provincia de Rio Negro) Relacto nes de la Sociedad Argentina de Antropologia Buenos Aires n 21 p 33481996

RADOVICH J BALAZOTE A Inversion y desinversion de capital en me gaproyectos hidroeneigeticos efectos sociales en poblaciones mapuches asentadas sobre los rios Limay y Neuquen Papeles de 7 rabajo Centro Inter disciplunario de Ciencias Etnolinguisticas y Antropologico Sociales Unı versidad Nacional de Rosanıo Rosarıo n 6 p 127142 nov 1997

RADOVICH J BALAZOTE A The forced resettlement process among the Mapuche population in Northern Patagonia Argentina Yumtzlob Tijds chrift over de Americas Rotterdam v 11 n 1,85 1011999

RADOVICH J BALAZOTE A Proyecto Mega disputa terntorial y recono cımiento etnico en Kaxipayiñ Relactones de la Sociedad Argentına de Antropo logia Buenos Aures n 26 p 107 117, 2000a

RADOVICH J BALAZOTE A Desinversion de capital y conflicto social los cortes de ruta en Cutral Co Paza Huincul Etnia Digital IIAO Olavarna, n 444520002002 
REHNFELDT Marilin Las tinieblas envuelven la Tierra la construccion de la hidroelectrica Yacyreta y la relocalızacion de los indigenas Mbya Guara nı del Mbaepu In SANTOS Sílvio Coelho dos, NACKE Aneliese (Org) Hidreletricas e povos indigenas Florianopolıs Letras Contemporâneas 2003 p 3766

REIS Marıa Jose Estado hıdreletricas e socıedade implıcações politıco ideologicas In CONGRESSO BRASILEIRO DE ECONOMIA E SOCIO LOGIA RURAL, 29 Campinas julho de 1991 Anats Campinas 1991 p 179208

REIS Maria Jose et al Hidreletricas e reassentamento compulsono de populações aspectos socıo culturass Curıtıba 1993 Trabalho realızado para o Instıtuto Ambıental de Parana julho de 1992 a março de 1993 Mimeo

REIS Mana Jose Espaços vivências e identıdade In MAGALHÃES S M BRITTO E de C CASTRO E R (Org) Energia na Amazônı Belem Mu seu Paraense Emılıo Goeldı Unıversıdade Federal de Para Assocıação das Universidades Amazônucas 1996

REIS Mana Jose Espaços vivências e identıdades os camponeses do Alto Uruguaı e a hidroeletrica de Ita In CONGRESO ARGENTINO DE AN TROPOLOGIA SOCIAL 5 Universıdad Nacional de La Plata I997 Ana les La Plata 1997

REIS Marıa Jose Espaços vividos migração compulsoria tdentıdade os campo neses do Alto Uruguaı e a hidreletrica de Ita Tese (Doutorado em Ciências Socıaıs) - Programa de Pos Graduação em Ciêncıas Socıaıs Unıversıdade Estadual de Campinas Campinas 1998

REIS Marıa Jose Espaços vivências e ıdentıdade os camponeses do Alto Uruguar e a hidrelétrica de Ita In BALAZOTE A CATULLO $M$ RA DOVICH J (Org ) Antropologia y grandes proyectos en el Mercosur La Plata Mmerva 2001a p 89106

REIS Maria Jose O reassentamento de produtorcs famllares o tempo da reconstrução e recriação dos espaços In REIS M J BLOEMER N M S (Org) Hidreletricas e populações locals Florianopolıs Cidade Futura Ed da UFSC 2001 b p 119166

REIS Marı Jose O reassentamento de pequenos produtores rurais refle xões sobre processos de reconstrução dos espaços fisıcos e sociaıs In FO RUM TECNICO DE REASSENTAMENTO INVOLUNTARIO 2002 Salva dor Anats Salvador 2002

REIS Marıa Jose Desafios frustrações e conquistas a retomada das atıvı dades produtivas em reassentamentos de produtores familiares In PAU LILO Marıa Ignez S SCHMIDT Wilson (Org) Agricultura e espaço rural em Santa Catarna Flonanopolıs Ed da UFSC 2003 p 125157

REIS Maria Jose, BLOEMER Neusa M Sens (Org) Hidreletricas e populaçōes locals Florianopolıs Cidade Futura Ed da UFSC 2001

REIS Maria Jose BLOEMER Neusa M Sens A eletricidade como suporte 
da modernudade no cotıdıano In REIS Marıa Jose SANTOS, Silvıo Coe lho dos (Org) Memoria do setor eletrico na Região Sul Florianopolıs Ed da UFSC 2002a p 7794

REIS Marıa Jose BLOEMER Neusa M Sens A energıa eletrıca na Regıão Sul no contexto da privatızação In REIS Mana Jose SANTOS Sılvıo Coelho dos (Org ) Memoria do setor eletrico na Regzão Sul Flonanopolıs Ed da UFSC 2002b p 213226

REIS Marıa Jose BORNHOLDT Luciano Campelo O poder de representar e de ampluar direitos o caso do Movimento de Atıngidos por Barragens (MAB) Seminarios de Pesquisa do Programa de Mestrado Profissionalizante em Gestão de Poltticas Publicas UNIVALI Itajai v 42003

REIS Marıa Jose CATULLO Marıa Rosa CASTELLS Alıcıa Patrımônı reassentamento compulsorio e turismo um estudo comparativo sobre Federacion (Argentina) e Ita (Brasil) Ilha Revista de Antropologia Depar tamento de Antropologia Unıversidade Federal de Santa Catarına Flo rianopolıs v 5 n 2 p 77922003

REIS Maria Jose NACKE Analiese BLOEMER Neusa M S Empreend1 mentos pıoneiros na produção de energıa eletrıca In REIS Marıa Jose SANTOS Sílvı Coelho dos (Oıg) Memoria do setor eletrico na Região Sul Florianopolis Ed da UFSC 2002 p 3176

REIS Marıa Jose SANTOS Silvio Coelho dos (Org) Memoria do setor eletrico na Região Sul Florianopolıs Ed da UFSC 2002

REIS Marra Jose SCHERER WARREN Ilse BLOEMER Neuza M Alto Uru gual migração forçada e reatuahzação da identıdade camponesa Travessia Revista do migrante Barragens, São Paulo v 2 n 6 p 2932 jan /abr 1990

RElS Marla Jose et al Hidreletricas e reassentamento compulsorio de popu lações aspectos socio culturaıs Curıtıba 1993 Trabalho realızado para o Instıtuto Ambiental de Parana julho de 1992 a março de 1993 Mimeo

SANTOS Silvio Coelho dos Os indios Xokleng memoria visual Florianopolis Ed da FURB, Ed da UFSC 1997

SANTOS Silvio Coelho dos Notas sobre hidreletricas e povos indigenas no Brasil In PRIETO Esther (Comp) Entre la resignacion y la esperanza Asuncion Intercontinental 1989

SANTOS Sulvio Coelho dos Peuples indigenes et developpement hydro electrique Ethntes Paris n II 12 p 56631990

SANTOS Sılvio Coelho dos A barragem de Ibuama e as populações atın gidas na area indigena documento denuncia Boletım de Ciências Sociats Flonanopolıs UFSC v 511991 a

SANTOS, Sílvio Coelho dos Projetos hidroeletricos e povos indigenas na Ama zônia In HEBETTE Jean $O$ cerco esta se fechando o impacto do grande capital na Amazônia Rio de Janeiro Vozes FASE NAEA UFPr 1991b p 7888

SANTOS Silvio Coelho dos NACKE, Analiese A Eletronorte e os projetos hidreletricos In HEBETTE Jean O cerco esta se fechando o impacto do grande capıtal na Amazônıa Rıo de Janelıo Vozes FASE NAEA UFPR $1991 \mathrm{p} 465 \mathrm{I}$ 
SANTOS Silvio Coelho dos Presas y cuestiones socio ambientales en el Bra sll Alteridades Iztapalapa UAM v 2 n 4 p 31371992 (Reacomodos y construccion de presas)

SANTOS Silvıo Coelho dos Metodologia para um estudo de projetos de de senvolvımento e suas implicações politıcas In ARANTES Antonı A et al (Org) Desenvolvimento e direitos humanos a responsabulidade do antro pologo Campinas Ed da Unicamp 1992 p 81102

SANTOS Sılvıo Coelho dos Lideranças indıgenas indıgenısmo oficial e des trução florestal o caso de Ibırama In MAGALHÃES Antonı C (Org) Soctedades indigenas e transformações ambıentais Belem NUMA, UFPa 1993 p 185203 Republicado no Anuario Antropologico Rio de Janeıro Tempo Brasileuro n 92 p 89-104 1993

SANTOS Silvio Coelho dos The dam of Ibirama and the Indians a tragedy in the South of Brazll In INTERNATIONAL CONGRESS OF AMERICAN ISTS 48 Indigenous Peoples and Dams Environmental/Social Impacts Estocolmo julho de 1994 Proceedings Estocolmo, 1994

SANTOS Sílvio Coelho dos Constitucion y violacion de los derechos de los pueblos indigenas en el Brasil In GRUNBERG G (Coord) Articulacion de la diversidad Quito Ecuador Biblioteca Abya Yala 1995 p 161174 Tercera Reunion de Barbados

SANTOS Sılvio Coelho dos Notas sobre o deslocamento de populações in digenas em consequêncıa da implantação de hıdroeletrıcas na Amazônıa In MAGALHÃES S M BRITTO E de C CASTRO E R (Org) Energia na Amazônı Belem Museu Paraense Emılı Goeldı Unıversıdade Fede ral de Para Associação das Unıversıdades Amazonıcas, 1996a

SANTOS Sılvio Coelho dos Lideranças indigenas e indıgenısmo ofıcial no Sul do Brasll Antropologı em Prımetra Mão Programa de Pos Graduação em Antropologia Social UFSC Florianopolıs n 10 1996b

SANTOS Sílvıo Coelho dos O homem do Sul In MONTEIRO Salvador KAZ Leonel Frontetra o Brasil mendional Rio de Janeiro Alumbramen to, 1996c p 235275

SANTOS Silvio Coelho dos FRIC a Liga Patriotica e os indios In CON GRESSO DE HISTORIA E GEOGRAFIA DE SANTA CATARINA 1996 Instıtuto Historıco e Geografico d€ Santa Catauna Flonanopolıs Anats Florlanopolıs IHGSC 1996d p 704714

SANTOS Sílvı Coelho dos Notas sobre a construção da antropologia no Brasil Honzontes Antropologicos Historias da Antropologia Porto Alegre v 3 n 7,p 62691997

SANTOS Sílvio Coelho dos Pueblos indigenas de Brasıl derechos constıtu cionales con tierras y luchas presentes In BARTOLOME $M$ BARABAS A (Coord) Autonomias etnicas y Estados nacionales Mexico DF Conaculta INAH 1998 p 215229

SANTOS Sılvio Coelho dos Hıdroeletrıcas e processo de prıvatızação no ce narıo brasıleıro Revista de Dıvulgação Cultural Florıanopolıs n 64 p 914 1998 
SANTOS Silvio Coelho dos NACKE Aneluese (Org) Hidreletricas e povos in digenas Florianopolıs Letras Contemporâneas 2003

SANTOS Sílvıo Coelho dos NACKE Anelıese A UHE bınacional Itaipu e os indıos do Oco1 In (Org) Hidreletricas e povos indigenas Florianopo lıs Letras Contempolâneas 2003 p 2136

SANTOS Sılvıo Coelho dos NACKE Anelıese A implantação da UHE Ma chadunho num cenarıo privatizado um caso para reflexivo In REIS Ma na Jose BLOEMER Neusa Marıa Sens (Org) Hidreletrucas e populaçóes locals Florianopolıs Cidade Futura Ed da UFSC 2001 p 7192

SANTOS Silvio Coelho dos HENRIQUES Karyn N R Hidroeletricas e o processo de privatızação no cenarıo brasıleuro In BALAZOTE Alejandro CATULLO Maria R RADOVICH Juan Carlos (Org) Antropologia y gran des proyectos en el Mercosur La Plata Minerva 2001 p 5768

SANTOS Sílvio Coelho dos et al Barragens e questões socio ambientais no Brasil In MAUES Raymundo H (Org) REUNIÃO REGIONAL DE AN TROPOLOGOS DO NORTE E NORDESTE 3 Belem 1996 Anats Belem 1996 v 1 p 1728 